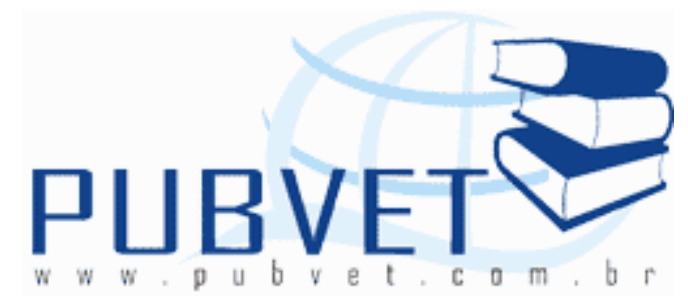

PUBVET, Publicações em Medicina Veterinária e Zootecnia.

\title{
Resistência ao anti-helmíntico levamisole em caprinos no Município de Grossos, Rio Grande do Norte, Brasil
}

Josivania Soares Pereira ${ }^{1}$, Saul Vitor Ferreira de Brito Oliveira², Wesley Adson Costa Coelho ${ }^{3}$, Zuliete Aliona Araújo de Souza Fonseca ${ }^{3}$, Weibson Paz Pinheiro André ${ }^{4}$, Sílvia Maria Mendes Ahid $^{5}$

${ }^{1}$ Bióloga, técnica do Laboratório de Parasitologia Animal e doutoranda do Programa de Pós-Graduação em Ciência Animal da Universidade Federal Rural do Semi-Árido (UFERSA). E- mail: josigej@ufersa.edu.br

${ }^{2}$ Médico veterinário.

${ }^{3}$ Médicos veterinário e doutorandos do Programa de Pós-Graduação em Ciência Animal da UFERSA.

${ }^{4}$ Discente da graduação em Medicina Veterinária da UFERSA.

${ }^{5}$ Médica veterinária, Docente da UFERSA.

\section{Resumo}

Apesar do surgimento de drogas anti-helmínticas de amplo espectro de ação, o controle dos nematódeos gastrintestinais vem sendo prejudicado pelo aparecimento da resistência parasitária. A presente pesquisa objetivou verificar a sensibilidade dos nematódeos gastrintestinais de caprinos a ação do vermífugo cloridrato de levamisol a $10 \%$. O experimento foi realizado em quatro criações de caprinos localizadas no município de Grossos, Rio Grande do Norte (RN). Em cada propriedade foram selecionados 30 animais, divididos em 
dois grupos de 15 animais: um tratamento com o cloridrato de levamisol a $10 \%$ e outro sem tratamento. No dia zero os animais foram vermifugados e 10 dias pós-tratamento fez-se a coleta de fezes e processamento das amostras por análises de OPG e Coprocultura. Strongyloides sp., o mais prevalente em todos os grupos tratados, apresentou percentual de helmintos resistentes ao levamisole em 75\%. Haemonchus contortus foi encontrado em $75 \%$ dos efetivos avaliados. A redução na contagem de ovos nas fezes 10 dias póstratamento com levamisole variou entre $27 \%$ e $97 \%$. Quanto a resistência ao cloridrato de levamisol a $10 \%$ observou-se que o Strongyloides sp. obteve maior índice de resistência, seguida por, Oesophagostomun sp., Trichostrongylus sp. e Haemonchus sp. O perfil das propriedades revelou que as drogas anti-helmínticas utilizadas pertenciam aos grupos dos benzimidazóis, avermectinas e imidazotiazois. Nestas propriedades não se adotavam manejo correto dos animais como também havia pelo criador, desconhecimento da dose correta e uso dos vermífugos.

Palavras-chave: Haemonchus contortus, Imidazotiazois, Strongyloides, Vermifugação.

\title{
Resistance to anthelmintic levamisole in goats in the city of Grossos, Rio Grande do Norte, Brazil
}

\begin{abstract}
Despite the emergence of anthelminthic drugs for broad spectrum of action, control of gastrointestinal nematodes has been hampered by the emergence of parasite resistance. This study aimed to verify the sensitivity of gastrointestinal nematodes of goats anthelmintic action of cloridrato de levamisol a $10 \%$. The experiment was conducted in four creations of goats in the county of Grossos, Rio Grande do Norte (RN). In each property we selected 30 animals were divided into two groups of 15 animals: a treatment with cloridrato de levamisol a $10 \%$ and another without treatment. On day zero, the animals were dewormed and 10 days post-treatment was done to collect stool samples for
\end{abstract}


PEREIRA, J.S. et al. Resistência ao anti-helmíntico levamisole em caprinos no Município de Grossos, Rio Grande do Norte, Brasil. PUBVET, Londrina, V. 7, N. 19, Ed. 242, Art. 1599, Outubro, 2013.

processing and analysis of OPG and faecal cultures. Strongyloides sp., the most prevalent in all treated groups, presented a percentage of helminths resistant to levamisole in $75 \%$. Haemonchus contortus was found in $75 \%$ of the assessed effective. The reduction in faecal egg counts 10 days after treatment with levamisole ranged between $27 \%$ and $97 \%$. As resistance to Cloridrato de levamisol a $10 \%$ observed that Strongyloides sp. had a higher resistance index, followed by, Oesophagostomun sp., Trichostrongylus sp. and Haemonchus sp. The profile of the properties revealed that anthelminthic drugs used were from the groups of the benzimidazoles, avermectins and imidazotiazois. These properties are not adopted proper handling of animals also had the farmer, unaware of the correct dosage and use of anthelmintics.

Keywords: Haemonchus contortus, Imidazotiazois, Strongyloides, Worming.

\section{Introdução}

A infecção por nematódeos gastrintestinais é considerada o principal problema sanitário da criação de pequenos ruminantes no Brasil (Igarashi et al., 2013). Sendo que estes últimos constituem a principal fonte protéica e de geração de renda dos agricultores e de suas famílias. Entretanto, alguns fatores, como exemplo as endoparasitoses, limitam a produção desses animais, especialmente nas regiões tropicais, onde os prejuízos econômicos são mais acentuados (Vieira, 2013).

No Brasil, a região Nordeste concentra os maiores rebanhos, dentre eles os caprinos representam 94\%. Nesta região, a caprinocultura é uma atividade econômica que já se encontra em bastante expansão e é considerada fonte de proteína de origem animal com importante relevância socioeconômica para quem vive dessa atividade (Lima et al., 2010).

Os caprinos criados na região semiárida podem ser parasitados por Haemconchus contortus e Trichostrongylus axei, que se localizam no abomaso, Trichostrongylus colubriformis, Strongyloides papillosus, Cooperia punctata, Cooperia pectinata e Bunostomum trigonocephalum, que parasitam o intestino 
delgado, e Oesophagostomum colubianum, Trichuris ovis, Trichuris globulosa e Skrjabinema sp. que vivem no intestino grosso (Costa et al., 2011). Levantamentos realizados no Nordeste brasileiro demonstraram que mais de $80 \%$ da carga parasitária de ovinos e caprinos está constituída por $H$. contortus (Arosemena et al., 1999).

O controle do parasitismo é feito, basicamente, com a utilização de antihelmínticos (Charles et al., 1989). Em caprinos quando falhas ocorrem nesse tipo de controle aparece a resistência anti-helmíntica definida como a capacidade de uma população de parasitos em sobreviver a doses de antihelmínticos que poderiam ser letais para populações susceptíveis (Vieira, 2008; Torres-Acosta \& Hoste 2008). Esta habilidade de sobreviver a futuras exposições de uma droga pode ser transmitida aos seus descendentes (Costa et al., 2011).

Pertencente ao grupo dos imidazotiazois, o anti-helmíntico cloridrato de levamisol a $10 \%$ possui atividade sobre estados adultos e imaturos em desenvolvimento de nematódeos gastrintestinais e pulmonares de ruminantes, equinos, suínos e aves. Não é ovicida, possui baixa atividade sobre Trichuris spp. e estágios imaturos inibidos de nematódeos e não tem ação sobre cestódeos e trematódeos (Spinosa, 2006).

Nesta linha, este trabalho teve por objetivo detectar a ocorrência de resistência ao anti-helmíntico cloridrato de levamisol a $10 \%$ em nematódeos gastrintestinais de caprinos no município de Grossos, Rio Grande do Norte (RN).

\section{Material e Métodos}

O experimento foi conduzido no município de Grossos, Rio Grande do Norte em criações tradicionais do sistema produtivo de caprinos da região geográfica Oeste. As análises da contagem de ovos por grama de fezes e coprocultura foram feitas no Laboratório de Parasitologia Animal da Universidade Federal Rural do Semi-Árido (UFERSA). 
PEREIRA, J.S. et al. Resistência ao anti-helmíntico levamisole em caprinos no Município de Grossos, Rio Grande do Norte, Brasil. PUBVET, Londrina, V. 7, N. 19, Ed. 242, Art. 1599, Outubro, 2013.

Realizou-se visitas a algumas propriedades rurais, as quais foram marcadas geograficamente por uso de GPS e cadastradas junto às associações organizadas de criadores de caprinos e ovinos no estado do RN, visando coleta de fezes e obtenção de informações mediante aplicação individual de questionários sobre os métodos antiparasitários usados. Para a seleção e inclusão dos animais no experimento obedeceu-se aos seguintes critérios: contagem de ovos por gramas de fezes (OPG), segundo recomendações de Parra et al. (2011) e a escolha de animais que não teriam sido tratados com qualquer droga anti-helmíntica por um período mínimo 90 dias.

Foram utilizados por propriedade testada 30 caprinos, com idade variada. Os animais, selecionados aleatoriamente, foram marcados individualmente por rebanho, em dois grupos de 15 animais cada: Grupo I que recebeu tratamento com anti-helmíntico à base de cloridrato de levamisol a $10 \%$ e o Grupo II, o controle sem o tratamento convencional. As doses utilizadas seguiram as recomendações do fabricante.

De cada caprino, naturalmente infectado por nematódeos gastrintestinais, foram coletadas amostras fecais diretamente da ampola retal, identificadas em sacos plásticos e mantidas sob refrigeração até a realização do OPG através da técnica de Gordon \& Withlock (1939), e obtenção das larvas por coprocultura quantitativa descrita por Ueno (1995). Sendo estas últimas identificadas de acordo com as características descritas por Keith (1953).

As médias aritméticas do número de ovos nas fezes, para cada grupo tratado (OPGt), foram calculadas e comparadas com as médias contadas no grupo controle (OPGc) e a redução da contagem de ovos nas fezes (RCOF) determinada pela fórmula descrita por Coles et al. (1992).

\section{RCOF $=[1-($ OPGt $/$ OPGC) $] \times 100$}

Em que:

RCOF $=$ teste de redução na contagem de ovos por grama de fezes 
OPGt= média do número de ovos por grama de fezes do grupo de animais tratados

OPGc = média do número de ovos por grama de fezes do grupo controle

O resultado positivo para resistência foram considerados à partir da percentagem de redução da contagem de ovos inferior a $95 \%$ e se o limite inferior do intervalo de confiança a $95 \%$ foi menor do que $90 \%$ (Molento, 2004; Prichard, 1990; Edwards et al., 1986). Os dados obtidos foram analisados pelo programa estatístico RESO (1989) o qual obedece as instruções WAAVP (Coles et al., 1992).

\section{Resultados e Discussão}

Do estudo das 23 propriedades abordadas, mediante uso individual dos questionários, foram selecionadas 4 propriedades para participar do experimento. As drogas anti-helmínticas mais utilizadas nas propriedades submetidas aos questionários pertenciam aos grupos dos benzimidazóis (BZD), avermectinas (AVM) e em menor escala imidazotiazois (IMZ). Destas, 56,5\% dos anti-helmínticos pertenciam apenas ao grupo das AVM; 39,1\% aos BZD e $4,3 \%$ aos IMZ.

Não foi evidenciado uso simultâneo de dois ou mais princípios ativos em uma mesma propriedade. Tais dados discordam de Vieira \& Cavalcante (1999), ao realizar um estudo em rebanhos caprinos no estado do Ceará verificaram que em $29,4 \%$ das propriedades os anti-helmínticos utilizados pertenciam ao grupo dos IMZ ou apenas dos BZD e algumas propriedades utilizavam, simultaneamente dois grupos de princípios ativos diferentes.

Com relação ao $H$. contortus, a população de helmintos apresentou sensibilidade frente ao cloridrato de levamisol a $10 \%$, em todas as propriedades em que este foi encontrado, divergindo de Vieira \& Cavalcante (1999) que ao realizarem estudos no estado do Ceará encontraram H.contortus resistente ao levamisol em $41,9 \%$ das propriedades estudadas. 
PEREIRA, J.S. et al. Resistência ao anti-helmíntico levamisole em caprinos no Município de Grossos, Rio Grande do Norte, Brasil. PUBVET, Londrina, V. 7, N. 19, Ed. 242, Art. 1599, Outubro, 2013.

A prevalência de Trichostrongylus sp. foi de $75 \%$ nas propriedades estudadas. O percentual de helmintos resistente ao cloridrato de levamisol a $10 \%$ atingiu a porcentagem de $25 \%$ das propriedades, o que concorda com Melo et al. (2003), que ao avaliar caprinos no estado Ceará encontrou para este mesmo helminto, resistência em $37,5 \%$ das propriedades estudadas, e também com Vieira \& Cavalcante (1999) que observaram resistência em $20,6 \%$ das propriedades.

Verificou-se Strongyloides sp. nas quatro propriedades analisadas, sendo o mais prevalente e com resistência de $75 \%$, o que diverge do observado por Lima et al. (2010) em Pernambuco e por Coelho (2009) em Mossoró, que encontraram $H$. contortus como mais prevalente.

De todas as propriedades analisadas, 75\% apresentaram resistência ao principio ativo testado, o que concorda com Vieira \& Cavalcante (1999) que ao avaliar a resistência ao levamisol no Ceará evidenciou tal situação em 73,5\% das propriedades analisadas.

Em ordem decrescente de resistência foi visto que a espécie Strongyloides sp. foi a que obteve maior índice de resistência, estando presente em $100 \%$ das propriedades estudadas, que, por sua vez, ofereceu resistência frente ao cloridrato de levamisol a $10 \%$ em $75 \%$ destas, seguida por Oesophagostomun sp., Trichostrongylus sp. e H. contortus (Tabela 1), o que discorda de Melo et al. (2003) e Morais (2007) que recuperaram somente larvas de Trichostrongylus sp.

Tabela 1 - Prevalência de nematódeos resistentes ao cloridrato de levamisol a $10 \%$.

Nematódeo No de Propriedades (\%)

Strongyloides sp.

Oesophagostomun sp.

$02(50)$

Trichostrongylus sp.

$01(25)$

Haemonchus contortus

$0(0,0)$ 
PEREIRA, J.S. et al. Resistência ao anti-helmíntico levamisole em caprinos no Município de Grossos, Rio Grande do Norte, Brasil. PUBVET, Londrina, V. 7, N. 19, Ed. 242, Art. 1599, Outubro, 2013.

Os dados do presente trabalho divergem ainda dos achados por Coelho (2009), que ao avaliar caprinos em Mossoró encontrou, respectivamente, Haemonchus sp., Trichostrongylus sp. e Oesophagostomun sp.

A redução na contagem de ovos nas fezes (RCOF) após o tratamento com cloridrato de levamisol a $10 \%$, variou entre $27 \%$ a $97 \%$ (Tabela 2 ). 0 percentual de redução diverge do encontrado por Vieira \& Cavalcante (1999) e Melo (2003), sendo que ao avaliar o percentual de RCOF obtiveram como resultado uma variação de 91,5 a $100 \%$ e 43 a $100 \%$, respectivamente.

Tabela 2- Redução na contagem de ovos nas fezes (RCOF) de caprinos tratados com cloridrato de levamisol a 10\%, 10 dias pós-vermifugação.

\begin{tabular}{llll}
\hline Propriedade & Controle & \multicolumn{2}{c}{ Levamisole } \\
\hline 1 & OPG médio* & OPG médio* & RCOF (\%) \\
2 & 1050 & 450 & 27 \\
3 & 1375 & 2208 & 97 \\
4 & 2041 & 1184 & 70 \\
\hline
\end{tabular}

* Média aritmética do número de ovos por grama de fezes dos animais de cada grupo.

Os resultados obtidos da cultura de larvas demonstraram que $100 \%$ das infecções eram por helmintos da superfamília Trichostrongyloidea e Rhabdiasoidea. Os nematódeos encontrados foram $H$.contortus, Strongyloides sp., Trichostrongylus sp. e Oesophagostomun sp. (Tabela 3) o que foi igualmente verificado por Ahid et al. (2008) ao avaliar os parasitos gastrintestinais de caprinos da região oeste do RN. A identificação das larvas infectantes mostrou que os nematódeos sobreviventes ao principio ativo testado foram principalmente Strongyloides sp., seguido pelo Oesophagostomun sp., divergindo de Ahid et al. (2007) em pesquisas feita em caprinos da zona da mata em Alagoas e de Coelho (2009), em Mossoró. 
PEREIRA, J.S. et al. Resistência ao anti-helmíntico levamisole em caprinos no Município de Grossos, Rio Grande do Norte, Brasil. PUBVET, Londrina, V. 7, N. 19, Ed. 242, Art. 1599, Outubro, 2013.

Tabela 3 - Composição da cultura de larvas, após 10 dias da administração do tratamento, em caprinos nas propriedades estudadas.

\begin{tabular}{cccccccccc}
\hline & \multicolumn{4}{c}{ Tratamentos } \\
\cline { 2 - 9 } Propriedades & \multicolumn{4}{c}{ Controle $(\%)$} & \multicolumn{4}{c}{ Levamisole $(\%)$} \\
& $H$ & $T$ & $O$ & $S$ & & $H$ & $T$ & $O$ & $S$ \\
\cline { 2 - 9 } & 0 & 0 & 0 & 100 & & 0 & 0 & 0 & 100 \\
2 & 15 & 6 & 1 & 78 & & 4 & 2 & 0 & 94 \\
3 & 19 & 2 & 5 & 74 & & 1 & 0 & 11 & 88 \\
4 & 36 & 2 & 10 & 52 & & 5 & 4 & 4 & 87 \\
\hline
\end{tabular}

$H=H$. contortus; $T=$ Trichostrongy/us sp; $O=$ Oesophagostomum sp; $S=$ Strongyloides sp.

No presente trabalho as coordenadas geográficas de cada propriedade evidenciaram nematódeos resistentes ao cloridrato de levamisol a $10 \%$ no percentual de 75\% (Tabela 4). A distribuição geográfica destas propriedades resistentes demonstrou-se presente na região noroeste da cidade de GrossosRN.

Tabela 4 - Coordenadas geográficas via satélite das 4 propriedades visitadas.

\begin{tabular}{ccc}
\hline Propriedades & \multicolumn{2}{c}{ Coordenadas } \\
\hline 1 & $4^{\circ} 51^{\prime} 49.58^{\prime \prime S}$ & $37^{\circ} 14^{\prime} 22.89^{\prime \prime O}$ \\
2 & $4^{\circ} 56^{\prime} 5.49 " \mathrm{~S}$ & $37^{\circ} 12^{\prime} 2.09^{\prime \prime} \mathrm{O}$ \\
3 & $4^{\circ} 56^{\prime} 53.21^{\prime \prime S}$ & $37^{\circ} 11^{\prime} 24.93^{\prime \prime} \mathrm{O}$ \\
4 & $4^{\circ} 51^{\prime} 49.58^{\prime \prime S}$ & $37^{\circ} 14^{\prime} 22.89^{\prime \prime O}$ \\
\hline
\end{tabular}

O perfil epidemiológico das propriedades resistentes no município de Grossos-RN revelou que, de acordo com os questionários, os criadores de caprinos adotavam um sistema de manejo animal ineficiente, como também evidenciavam um despreparo e desconhecimento do uso e dosagem correta dos anti-helmínticos utilizando em diversas ocasiões sub e superdosagem dos medicamentos. Os proprietários vermifugavam todo o rebanho sem respeitar um intervalo considerável de dias entre uma administração e outra. Evidenciou-se o uso de um mesmo grupo de princípio ativo por longos intervalos de tempo. 
PEREIRA, J.S. et al. Resistência ao anti-helmíntico levamisole em caprinos no Município de Grossos, Rio Grande do Norte, Brasil. PUBVET, Londrina, V. 7, N. 19, Ed. 242, Art. 1599, Outubro, 2013.

\section{Conclusões}

Os nematódeos gastrintestinais de caprinos provenientes das propriedades estudadas no presente trabalho e localizadas no município de Grossos-RN apresentaram resistência ao anti-helmíntico cloridrato de levamisol a $10 \%$.

\section{Referências}

Ahid S.M.M., Cavalcante M.D.A., Suassuna A.C.D., Soares H.S., Pereira R.H.M.A. 2007. Eficácia anti-helmíntica em rebanho caprino no Estado de Alagoas, Brasil. Acta Veterinária Brasílica.1: 56-59.

Ahid S.M.M., Suassuna A.C.D., Maia M.B., Costa V.M.M., Soares H.S. 2008. Parasitos gastrintestinais em caprinos e ovinos da região Oeste do Rio Grande do Norte, Brasil. Ciência Animal Brasileira. 9: 212-218.

ANUALPEC - Anuário da Pecuária Brasileira, 2006. São Paulo: FNP Consultoria \& Comércio. p.369.

Arosemena N.A.E., Bevilaqua C.M.L., Melo A.C.F.L., Girao M.D. 1999. Seasonal variations of gastrointestinal nematodes in sheep and goats from semi-arid áreas in Brazil. Revue Medicine Veterinaire. 150: 873-87.

Charles T.P., Pompeu J., Miranda D.B. 1989. Efficacy of three broad-spectrum anthelmintics against gastrointestinal nematode infections of goats. Veterinary Parasitology. 34: 71-75.

Coelho W.A.C. 2009. Resistência anti-helmíntica em caprinos no município de MossoróRN. Dissertação de mestrado, Universidade Federal Rural do Semi-Árido, Mossoró 37p.

Coles G.C., Bauer C., Borgsteede F.H.M., Geerts S., Klei T.R., Taylor M.A., Waller P.J. 1992. World association for the advancement of veterinary parasitology (WAAVP) methods for the detection of anthelmintic resistance in nematodes of veterinary importance. Veterinary Parasitology. 44: 35-44.

Costa, V.M.M.; Simões, S.V.D.; Riet-Correa, F. 2011. Controle das parasitoses gastrintestinais em ovinos e caprinos na região semiárida do Nordeste do Brasil. Pesquisa Vetetrinária Brasileira. 31: 65-71.

Edwards J.R., Wroth R., Chaneet G.C., Besier R. B., Karlsson J., Morcombe P.W., DaltonMorgan G., Roberts D. 1986. Survey of anthelmintics resistance in western Australia sheep flocks, prevalence. Australian Veterinary Jornal. 63:135-138.

Gordon H. M, Whitlock H.V. 1939. A new technique for couting nematode eggs in sheep faeces. Journal of the Commonwealth Scientific and Industrial Research Organization. 12: 50-52. 
PEREIRA, J.S. et al. Resistência ao anti-helmíntico levamisole em caprinos no Município de Grossos, Rio Grande do Norte, Brasil. PUBVET, Londrina, V. 7, N. 19, Ed. 242, Art. 1599, Outubro, 2013.

Keith R.K. 1953. The differential of the infective larval of some common nematode parasites of cattle. Australian Journal Zoollogy. 2: 223-230.

Igarashi M., Carvalho D.M.G., Bucci F.C., Miranda Y., Rodrigues Z.M., Almeida M.C.F. Piona M.N.M. Efeito do neem (Azadirachta Indica) no controle de nematódeos gastrintestinais em ovinos suplementados a pasto no período seco. 2013. Ciências Agrárias. 34: 301-310.

Lima M.M., Farias M.P.O., Romeiro E.T., Ferreira D.R.A., Alves L.C., Faustino M.A.G. 2010. Eficácia da moxidectina, ivermectina e albendazole contra helmintos gastrintestinais em propriedades de criação caprina e ovina no estado de Pernambuco. Ciência Animal Brasileira. 11: 94-100.

Lima W.C., Athayde A.C.R., Medeiros G.R., Lima D.A.S.D., Borburema J.B., Santos E.M., Vilela V.L.R., Azevedo S.S. 2010. Nematódeos resistentes a alguns anti-helmínticos em rebanhos caprinos no Cariri Paraibano. Pesquisa Veterinária Brasileira. 30: 1003-1009.

Magona J.W., Musisi G. 2002. Influence of age, grazing system, season and agroclimatic zone on the prevalence and intensity of gastrointestinal strongylosis in ugandan goats. Small Ruminant Research. 44: 187-192.

Melo A.C.F.L. 2005. Abordagem genética da resistência anti-helmíntica em Haemonchus contortus. Revista Portuguesa de Ciências Veterinárias. 100: 141-146.

Melo A.C.F.L., Reis I. F., Bevilaqua C.M.L., Vieira L.S., Echevarria F.A.M., Melo L.M. 2003. Nematódeos resistentes a anti-helmínticos em rebanhos de ovinos e caprinos do estado do Ceará, Brasil. Ciência Rural. 33: 339-344.

Morais F.R. 2007. Resistência anti-helmíntica em rebanhos ovinos da região da Associação dos Municípios do Alto Irani (AMAI). Ciência Animal Brasileira. 8: 559-565.

Molento M.B. 2004. Resistência de helmintos em ovinos e caprinos. Revista Brasileira de. Parasitologia Veterinária. 13: 82-85.

Parra C.L.C., Olivo C.J., Flores F.S., Agnolin C.A.P.C.C., Bolzan A.M.S. 2011. Alteração da carga de endoparasitas em ovinos submetidos a diferentes níveis de folha de bananeira na alimentação. Revista Brasileira de Agroecologia, 6(2): 111-116.

Prichard R.K. 1990. Biochemistry of anthelmintic resistance. Round Table Conf. Anais. International Congress of Parasitology, Paris. CD-ROM.

Sangster N.C. 2001. Managing parasiticide resistance. Veterinary Parasitology. 98: 89-109.

Spinosa H. S., Gorniak S.M. 2006. Farmacologia aplicada a medicina veterinária. 4a ed. Roca, Rio de Janeiro, 545 p.

Torres-Acosta, J.F.J.; Hoste, H. Alternative or improved methods to limit gastro-intestinal parasitism in grazing sheep and goats. 2008. Small Ruminant Research. 77: 159-173.

Ueno H. 1995. Cultivo quantitativo de larvas de nematódeos gastrintestinais de ruminantes com tentativa para pré-diagnóstico. International Cooperation Agency, Tokyo: Japão, p.138.

Vieira L.S. 2013. Fitoterápicos no controle de endoparasitoses de caprinos e ovinos. Capturado em 27 de mar. 2013. Online. Disponível na Internet http://periodicos.ufersa.edu.br/revistas 
PEREIRA, J.S. et al. Resistência ao anti-helmíntico levamisole em caprinos no Município de Grossos, Rio Grande do Norte, Brasil. PUBVET, Londrina, V. 7, N. 19, Ed. 242, Art. 1599, Outubro, 2013.

Vieira L.S.; Lôbo R.N.B; Cavalcante A.C.R.; Neves M.R.M; Navarro A.M.C.; Benvenuti C.L.; Zaros L.G. 2009. Panorama mundial dos métodos de controle de endoparasitoses. $4^{a}$ ed. Sincorte, João Pessoa.

Vieira L.S. 2008. Métodos alternativos de controle de nematódeos gastrintestinais em caprinos e ovinos. Revista Tecnologia \& Ciência Agropecuária. 2: 28-31.

Vieira L.S., Cavalcante A.C.R. 1999. Resistência anti-helmíntica em rebanhos caprinos no estado do Ceará. Pesquisa Veterinária Brasileira.19: 99-103. 